\section{Debating PD transplants}

\section{By Michael J. Haas, Senior Writer}

Clinicians have been transplanting dopaminergic cells into patients with Parkinson's disease for about 20 years, but they haven't followed exactly what happens to those implants for any longer than four years after surgery. ${ }^{1-4}$ Three papers in Nature Medicine now show the longerterm fate of those cells, based on results of postmortem histological analyses in people with Parkinson's disease who died of causes unrelated to the disease. ${ }^{5-7}$

Two of the papers show evidence of PD-associated aggregates in a small fraction of the transplanted cells, whereas the third does not. The issue at hand is how to explain those results and whether different types of transplants could avoid the problem of aggregate accumulation altogether.

Collectively the three studies examined transplants in 8 patients who passed away 9-16 years after receiving dopaminergic cell grafts transplanted from fetal midbrains.

Though its underlying cause is unknown, PD is characterized by protein aggregates that accumulate in, and eventually kill, dopaminergic neurons over the course of years or decades. Current therapies aim to counteract the resulting dopamine deficiency-for example, by using 3,4-dihydroxy-L-phenylalanine (L-dopa) - which can reverse symptoms and slow, but not halt, disease progression. ${ }^{8}$

Among the alternative treatments developed in the past two decades are cell-based therapies in which functional, dopamine-producing neurons are surgically implanted in the brains of people with PD, thereby compensating for the dysfunctional neurons. Such therapies deliver longer-term symptomatic relief than drugs like L-dopa.

But until now, it was not known how many of the transplanted cells continued to produce dopamine-or for how long.

The main molecular culprit in PD is $\alpha$-synuclein, a protein that occurs naturally in neural tissue in a soluble form. In PD, $\alpha$-synuclein becomes insoluble and precipitates inside neurons, where it associates with ubiquitin and other proteins to form aggregates called Lewy bodies. The proteasome cannot degrade Lewy bodies, so they accumulate in the neuron, where they impede the cell's normal functions and eventually kill it.

The presence of Lewy bodies in neurons, as indicated by histological analysis, is indicative of PD but not conclusive. That's because Lewy bodies occur in other, nonparkinsonian forms of dementia.

A European research team at Lund University Hospital, Wallenberg Neuroscience Center and University College London found
Lewy bodies in $2-5 \%$ of the neurons transplanted into 2 patients, one who had survived for 11 years and the other for 16 years after surgery. ${ }^{5}$ The team was led by Patrik Brundin, professor of neurobiology and group leader of the Neuronal Survival Unit at Wallenberg.

A U.S. research team at Mount Sinai School of Medicine, Rush University Medical Center and the University of South Florida found Lewy body-like pathology in the transplanted neurons of a single patient who had survived for 14 years after surgery. ${ }^{6}$ The team was led by Jeffrey Kordower, professor of neurosurgery and director of the Research Center for Brain Repair at Rush.

Finally, a North American team studied 5 patients who had survived 9-14 years after transplant surgery and found no signs of pathology in the grafts. ${ }^{7}$ This team included researchers from Dalhousie University, Queen Elizabeth II Health Science Centre, McGill University, McConnell Brain Imaging Centre, Montreal Neurological Institute, Harvard University and McLean Hospital, and the University of Pennsylvania. The team was led by Ole Isacson, professor of neurology and director of Neuroregeneration Laboratories and the Center for Neuroregeneration Research at Harvard and McLean.

\section{Habeas corpus}

The Brundin and Kordower teams both saw aggregates resembling Lewy bodies in their patients' transplants, leading the teams to suggest that PD had spread from the host brain to the fetal midbrain grafts.

A key question is whether the pathology was caused by disease propagation from host to graft or by variations between the transplant methods. The answer could lead to improvements in cell-based therapies and perhaps illuminate the mechanisms behind PD.

The Kordower team wrote that "our results suggest that grafted cells can be affected by the disease process and thereby might limit the long-term clinical benefit of these treatment approaches," including stem cell grafts.

"I'm very convinced there is a Parkinson's-like pathology in the dopamine cell transplants," Brundin told SciBX.

Kordower agreed. "Our data and Brundin's are virtually identical. Both show that transplants undergo Parkinson's disease-like changes."

Kordower also said the findings of both teams settle a long-running debate among PD researchers. The data, he said, show that the disease results from an ongoing process that continues to affect normal neurons throughout life and not from an acute insult to brain tissue that leads to long-term degeneration.

Marc Rubin, president and CEO of Titan Pharmaceuticals Inc., agreed with Brundin and Kordower. "Basically we're seeing that the disease process - whatever its pathophysiology_is ongoing and affects fetal midbrain implants. It could conceivably affect implants derived from stem cells in the same way."

Titan is developing a cell-transplant therapy that uses human retinal pigmented epithelial cells (RPEs) as "dopamine microfactories in the brain.” Because RPEs are not neural cells, they don't make connections to other neurons-and so should not be susceptible to the PD process, he said. 
The therapy is in a Phase IIb trial with partner Bayer Schering Pharma AG, a subsidiary of Bayer AG, with data expected this year.

Conversely, the Isacson team found no pathology in their patients' transplants and wrote that their results indicate that "the host brain does not necessarily create conditions that cause Parkinson's disease-related neurodegeneration in the transplanted neurons."

Curt Freed, professor and head of clinical pharmacology and toxicology and director of the Neurotransplantation Program for Parkinson's Disease at the University of Colorado School of Medicine, said his experience matched Isacson's results.

"I believe that we have done the largest series of dopamine cell transplants in the world-a total of 61 patients since 1988-and we have seen no protein deposits in dopamine neurons up to 14 years after transplant. Since the mechanism by which Parkinson's develops is unknown in most patients, and because the precipitation of $\alpha$-synuclein and ubiquitin proteins is not unique to Parkinson's," there is no reason to attribute the observed pathology to Parkinson's disease progression, he said.

Freed said the results of his study will be reported in a forthcoming publication.

Brundin acknowledged that there are other explanations besides PD for the pathology his team observed. "You could play devil's advocate and say that the pathology is not related to Parkinson's disease, that it could be related to aging," he said. "We don't have controls in the usual sense, so we don't know whether we would see the same pathology in normal patients after 16 years. But I do see a potential causal link because in these cases we already know the patients have Parkinson's disease-so we have to consider disease propagation a strong possibility."

Brundin offered an alternative explanation for the difference between studies.

"The number of cells that had survived in the 14-year patient [in Isacson's study] was about 9,800," Brundin said. "Kordower and our group had access to 10 to 15 times as many cells, because the transplant sizes were larger. In our studies, the proportion of dopaminergic transplant cells having Lewy bodies was between roughly $2 \%$ and $5 \%$. When you apply this to 9,800 cells, I think Isacson's team may have just missed spotting the Lewy bodies."

\section{Inflammatory reactions}

In contrast, both Freed and Isacson attribute the pathology seen by the Brundin and Kordower teams to differences in transplant methods. Fetal midbrain cells can be transplanted as solid pieces, as cell suspensions or as tissue strands.

All the patients studied by Isacson's team received suspensions of midbrain cells. The patients in the Kordower and Brundin studies received solid pieces of midbrain.

Isacson said his team thinks solid-piece transplants induce an immune response, as indicated by earlier studies that found accumulation of microglia - the brain's immune cells-in such grafts. ${ }^{2,3}$ Those studies were conducted by Freed and by Kordower team member C.
Warren Olanow, professor and chairman of neurology and professor of neuroscience at Mount Sinai.

The latest Kordower team wrote in Nature Medicine that they observed microglial activation in their patient's graft "to a degree that far exceeded the expression of microglia" in the surrounding host tissue. The Brundin team observed some microglia accumulation in their two patients' grafts, but wrote that the "immunohistochemistry did not indicate strong activation."

Isacson's team saw little indication of a microglial reaction to the transplanted cell suspensions. Thus, his group suggested that extracellular matrix and other non-neural tissue in solid pieces induce a chronic host inflammatory response that results in degeneration of the implanted cells over time.

"Individual differences in chronic inflammatory reactions around the implanted cells that are patient- or method-specific variations have been found, which could account for differences in protein aggregates" noted among the three papers, Isacson said. Current and future studies should determine how best to minimize inflammatory response, he added.

Michel Levesque, president and CEO of NeuroGeneration Inc., agreed with Isacson. "The presence of microglia indicates an immune reaction to the grafts that caused cell dysfunction and initiated the formation of Lewy bodies," he said.

- Marc Rubin,

He also said the grafts develop pathology in the same way as the original host cells do-as a result of "life-long exposure of the cells to oxidative stressors," which could include chemicals known to cause PD-like pathology.

"I think that the immune reaction and exposure to oxidative stressors are two different mechanisms that both induce upregulation of $\alpha$-synuclein, and their effect is additive," Levesque said.

NeuroGeneration is developing a transplant therapy for PD that uses neurons derived from the patient's own neural stem cells. According to Levesque, the implanted cells are a mixture of dopaminergic neurons and neurons that produce other neurotransmitters, such as $\gamma$-aminobutyric acid (GABA). Using a patient's own cells should avoid immunogenicity issues, he said.

Levesque said the company's Phase II trial of its neural stem cell therapy is on hold awaiting the completion of a GMP-compliant cell processing facility and should resume within six months.

Erik Miljan, head of stem cell discovery at ReNeuron plc, said the quality of the cell material used also could affect whether transplanted cells develop pathology.

In the three papers, "it is generally assumed that primary tissue isolated directly from fetal donor is the highest potency because it is from a developing brain and not manipulated," he said. "However, uncontrolled variables arise-for example, when multiple donors are used for one patient and the tissues must be stored until enough is collected."

Ideally, the potency and efficacy of the cells for implantation should be assessed by in vitro assays and in vivo studies in animal models. "Unfortunately, the limited amount of material that is available from a 
fetal donor frequently precludes such testing because all of the material is required for the treatment," he said.

Freed said his group has incorporated this kind of assessment into their transplantation method. "We collect tissue up to one month before transplant, holding it in tissue culture so that we can make sure the tissue is not infected and that it is making dopamine," he said. "No other group does this testing. The method also gives us time to accumulate multiple samples and schedule surgery as an elective-rather than emergency-surgery."

ReNeuron has human stem cell lines

\section{"Now we have to} understand why and how Lewy bodies form in grafts."

-Patrick Brundin,

Wallenberg Neuroscience Center
Nevertheless, Kordower noted "the vast majority of transplanted cells survive. So yes, this means that stem cell research should go forward."

Kordower sits on the scientific advisory board of Ceregene Inc., which has CERE-120, an adeno-associated virus type 2 vector encoding the neurturin (NTN) gene, in Phase II testing for PD with partner Genzyme Corp.

"Overall evidence points to robust, longterm survival of implants in all studies," Isacson said. He said his Nature Medicine coauthor Ivar Mendez-who is head of neurosurgery at both Dalhousie University and Queen Elizabeth II Health Sciences Centre-is leading a team to (ReN004) in discovery research for PD. Miljan anticipates that such cell lines would provide a continuous, renewable and standardized source of dopaminergic cells.

\section{Troubles in paradigm?}

One question at least one team plans to tackle is how the Lewy bodies show up in the transplanted cells.

"Now we have to understand why and how Lewy bodies form in grafts," Brundin said. "Knowing the mechanism could help us to design stem cell-derived grafts that are less prone to Lewy body formation."

He added: "Perhaps an understanding of the molecular basis for the host-to-graft propagation of pathology could also help us devise therapies to slow progression of the disease in patients without transplants."

Brundin said his team is planning in vitro and in vivo studies to investigate the mechanism by which $\mathrm{PD}$ pathology propagates from host to graft cells.

NeuroGeneration's Levesque noted that "not much is known about the brain's immune response to implants. The findings [in the three papers] suggest that current cell-based therapies may not be the best methods" because any cell source foreign to the host will be immunogenic. This is why NeuroGeneration is developing autologous stem cell therapies, he said.

Levesque also thinks the medical community and industry operate from a PD paradigm that is too narrow and could therefore limit researchers' ability to explain the differences between the three Nature Medicine papers. The PD community has "simplified Parkinson's disease to the extreme by describing it as a dopamine deficiency," he said.

"We have to approach the disease differently, with an understanding of all of the modified neurotransmitters in Parkinson's disease-not just dopamine-and how these modifications change the brain," he said.

For instance, he noted L-dopa is thought to create an imbalance between dopamine and GABA in the dopamine-deficient brain. "The chronic imbalance leads to dyskinesia and motor function complications resulting from the drug itself,' he said. He added that chronic intake of drugs like L-dopa is also suspected to cause damage to neurons.

Cell-based therapies, like NeuroGeneration's, that produce a mixture of neurotransmitters should do a better job of treating the whole disease process than do therapies that focus only on restoring dopamine production, Levesque said. develop new methods of preparing fetal cells, surgical improvements and stem cell-derived dopaminergic neurons.

"I find it remarkable that all three Nature Medicine reports, and our experience in Colorado, show that dopaminergic cell transplants survive and function indefinitely despite the differences in methods," Freed said. "This is a positive, not a cautionary, story."

\section{REFERENCES}

1. Piccini, P. et al. Nat. Neurosci. 2, 1137-1140 (1999)

2. Freed, C. et al. N. Engl. J. Med. 344, 710-719 (2001)

3. Olanow, C. et al. Ann. Neurol. 54, 403-414 (2003)

4. Mendez, l. et al. Brain 128, 1498-1510 (2005)

5. Li., J.-Y. et al. Nat. Med.; published online April 6, 2008; doi:10.1038/nm1746

Contact: Patrik Brundin, Wallenberg Neuroscience Center, Lund, Sweden

6. Kordower, J. et al. Nat. Med.; published online April 6, 2008; doi:10.1038/nm1747

Contact: Jeffrey H. Kordower, Rush University Medical Center, Chicago, III.

e-mail: jkordowe@rush.edu

7. Mendez, I. et al. Nat. Med.; published online April 6, 2008; doi:10.1038/nm1752

Contact: Ole Isacson, Harvard University and McLean Hospital, Belmont, Mass.

e-mail: isacson@hms.harvard.edu

8. Edelson, S. SciBX 1(8), 7-8; March 20, 2008

COMPANIES AND INSTITUTIONS MENTIONED

Bayer AG (Xetra:BAY), Leverkusen, Germany

Dalhousie University, Halifax, Nova Scotia, Canada

Genzyme Corp. (NASDAQ:GENZ), Cambridge, Mass.

Harvard University, Belmont, Mass.

Lund University Hospital, Lund, Sweden

McConnell Brain Imaging Centre, Montreal, Quebec, Canada

McGill University, Montreal, Quebec, Canada

McLean Hospital, Belmont, Mass.

Montreal Neurological Institute, Montreal, Quebec, Canada

Mount Sinai School of Medicine, New York, N.Y.

Queen Elizabeth II Health Science Centre, Halifax, Nova Scotia,

Canada

NeuroGeneration Inc., Los Angeles, Calif.

ReNeuron plc (LSE:RENE), Guildford, U.K.

Rush University Medical Center, Chicago, III.

Titan Pharmaceuticals Inc. (AMEX:TTP), South San Francisco, Calif.

University College London, London, U.K.

University of Colorado School of Medicine, Denver, Colo.

University of Pennsylvania, Philadelphia, $\mathrm{Pa}$.

University of South Florida, Tampa, Fla.

Wallenberg Neuroscience Center, Lund, Sweden e-mail: patrik.brundin@med.lu.se

Ceregene Inc., San Diego, Calif. 